\title{
Le bénéfice du protocole ERAS dans les suites opératoires de la cystectomie radicale: Analyse d'une série marocaine monocentrique
}

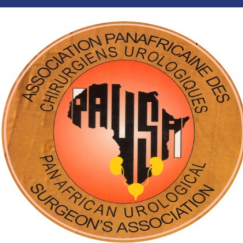

\author{
Othmane Yddoussalah ${ }^{1}$, Amine Saouli, Imad Ziouziou ${ }^{2}$, Tarik Karmouni ${ }^{1}$,Khalid El Khader', \\ Abdellatif Koutani ${ }^{1}$, Ahmed Iben Attya Andaloussi ${ }^{1}$
}

${ }^{I}$ Service d'urologie B, CHU Ibn Sina, Faculté de médecine et de pharmacie, Université Mohamed V, Rabat, Morocco, ${ }^{2}$ Service d'urologie, Faculté de médecine et de pharmacie, Université Ibn Zohr, Agadir, Morocco.

\section{Résumé}

Objectifs: Le but de cette étude est d'évaluer l'impact de l'implementation du protocole ERAS après cystectomie radicale pour tumeur de vessie.

Matériel et méthodes: Il s'agit d'une étude prospective monocentrique comparative d'un groupe témoin avec un protocole standard regroupant les cystectomies opérées pour tumeur de vessie de Mars 2017 en Avril 2018, et un groupe ERAS. Les critères de jugement évalués étaient les délais de reprise de gaz, de la première défécation, la durée du séjour hospitalier.

Résultats: Au total, 45 patients ont été inclus, 18 patients témoin et 27 patients ERAS. La moyenne du séjour hospitalier était de $9.4 \mathrm{j}$ vs $11.7 \mathrm{j} ; p=0.05$ en faveur du groupe ERAS. Le délai de reprise de gaz en heures était plus élevé dans le groupe témoin (69.8 vs 36.4; $p=<0.0001$ ). Le délai de la première défécation en heures était moindre dans le groupe ERAS (126.6 vs 64.6; $p=<0.0001)$. Le taux de complications postopératoires générales a été $31 \%$ dans le groupe témoin et de $24.4 \%$ dans le groupe ERAS.

Conclusion: l'application du protocole ERAS a permis de diminuer la durée du séjour hospitalier et a tendance à réduire le taux d'iléus postopératoire.

Mots clés: RAAC; cystectomie; suites opératoires.

Rec, u le: 23 June 2020, Accepté le: 31 August 2020

Auteur correspondant: Amine Saouli, Service d'urologie B, CHU Ibn Sina, Faculté de Médecine et de Pharmacie, Université Mohamed V, Rabat, Morocco, Email: amine.saouli0@gmail.com

ISSN: 2090-2379, https://afju.journals.ekb.eg

\section{Introduction}

La cystectomie radicale pour tumeur de la vessie est une chirurgie considérée longtemps comme une chirurgie majeure pourvue d'une morbi-mortalité non négligeable.

Durant la dernière décennie, la codification de cette technique chirurgicale a permis de réduire la mortalité moins de $5 \%{ }^{[1-3]}$. Néanmoins, la morbidité reste augmentée variant de 30 à $60 \%$ selon les études avec une durée moyenne du séjour hospitalier comprise entre 8 et 22 jours $^{[3-5]}$.

Le programme enhanced recovery after surgery (ERAS), est une approche multidisciplinaire qui rassemble plusieurs recommandations dans la phase pré-, per- et postopératoire ciblant une diminution des complications périopératoires et de la durée de séjour. Son efficacité a été démontrée initialement en chirurgie colorectale.
Le but de cette étude est d'évaluer l'impact de l'implémentation du programme ERAS sur la durée du séjour hospitalier et le taux de complications générales et gastro-intestinales ainsi que le délai de reprise de gaz et de la première défécation.

\section{Matériels et méthodes}

Il s'agit d'une étude prospective monocentrique randomisée, comparant les résultats postopératoires d'une série de patients suivant le nouveau programme de réhabilitation (ERAS) et d'un groupe témoin suivant le protocole standard, composé de patients opérés dans le service d'urologie B de l'hôpital Ibn Sina de Rabat du Mars 2017 en Avril 2018. Étaient inclus dans l'étude l'ensemble des cystectomies radicales avec curage 
ganglionnaire et dérivations urinaires non continentes de type Bricker et urétérostomie cutanée directe et dérivations urinaires continentes pour cancer de vessie. Étaient exclus les patients ayant eu une cystectomie pour vessie neurologique ou autre cause dans le but d'homogénéiser au maximum les groupes et limiter les biais de sélection.

Une information orale, claire et appropriée a été donnée à tous les patients inclus, après remise d'une fiche d'information détaillée sur la chirurgie et le déroulement du programme ERAS.

\section{Déroulement de l'étude et modalité du protocole «ERAS UROLOGIE B»:}

\section{a. Période préopératoire}

-Informations au patient: Lepatientavaitune information orale et écrite sur le déroulement de l'hospitalisation et les modalités du protocole de réhabilitation améliorée. Les patients ont été informés des avantages de ce protocole mais aussi des risques de complications et du déroulement de la convalescence.

-Sevrage de la consommation alcoolique et tabagique

-Jeûne préopératoire: Un jeûne de 2 heures pour les liquides clairs et $8 \mathrm{~h}$ pour les solides est suffisant avant l'induction de l'anesthésie générale.

-Apport de solutions (d'hydrates de carbone) sucrées orales : La prise d'une solution glucidique huit heures et deux heures avant l'intervention est recommandée. La dose de charge en glucides préconisée est 2 cuillères-à-café de sucre dissous dans du liquide clair.

-Pas de préparation intestinale sélective: seulement un lavement rectal durant la veille de la chirurgie.

-Thromboprophylaxie: Le patient reçoit une thromboprophylaxie avec une héparine de bas poids moléculaire. Le traitement est débutée le soir de l'intervention.

-Antibioprophylaxie:

Le patient reçoit une antibioprophylaxie débutée dans l'heure précédent le début de la chirurgie.

\section{b. Période peropératoire}

-Protocole anesthésique:

- Agents anesthésiques à action courte.

- Maintien de la volémie
-Sonde gastrique: $\mathrm{Si}$ une sonde d'aspiration nasogastrique est mise en peropératoire, elle est retirée en salle d'opération de manière systématique en fin d'intervention.

-Drainage abdominal: mettre en place le moins de drain possible, éviter le drain de la loge vésicale qui n'est pas indispensable.

\section{c. Période postopératoire}

-Pas de nutrition parentérale

-Utilisation d'agents prokinétiques: métoclopramide 10 $\mathrm{mg}$ toutes les $8 \mathrm{~h}$ (systématiquement), jusqu'à avoir transit positif des gaz.

-Mastication de chewing- gum (à raison de 3 fois 15 minutes par jour)

-Analgésie postopératoire:

Antalgiques non morphiniques:

Ils seront débutés en fin d'intervention: Paracétamol (sauf contre-indication) par voie intraveineuse puis orale dès que possible.

-Apport nutritionnel:

Le patient est encouragé à se réalimenter dès la 6ème heure après l'intervention, en commençant par une alimentation liquide de faible volume (300500- cc). Au deuxième jour postopératoire, les patients étaient autorisés une alimentation légère. En fonction de la tolérance, une alimentation normale devrait être obtenue progressivement en postopératoire.

-Mobilisation précoce:

Le patient est pris en charge par une équipe paramédicale l'encourageant à se mobiliser et à devenir indépendant.

\section{d. Protocole standard}

Alors que l'ancien protocole était basé essentiellement sur le jeune prolongé préopératoire, la préparation digestive, le maintien de la sonde nasogastrique en postopératoire, le drainage abdominal prolongé, l'utilisation des analgésiques morphiniques, l'apport systématique de la nutrition parentérale, et le retard de reprise d'alimentation.

\section{Analyse Statistique}

L'étude statistique a été réalisée sur le logiciel SPSS Statistics (version 20. IBM). Les variables quantitatives sont exprimées en médiane, avec écart interquartile. 
Les valeurs qualitatives sont exprimées en pourcentage.

L'analyse univariée a été réalisée en utilisant le test de chi-2 ou de Fisher comme approprié. Pour les variables quantitatives, on a utilisé le test statistique de student.
Le résultat a été considéré comme statistiquement significatif lorsque $p<0.05$.

\section{Résultats}

Les 2 groupes étaient comparables en termes de données démographiques et fonctionnelles (Tableau 1,2).

Tableau 1: Les variables qualitatives des patients de deux protocoles de notre série

\begin{tabular}{|c|c|c|c|c|}
\hline & \multicolumn{2}{|c|}{ Protocole } & \multirow[t]{2}{*}{ Total } & \multirow[t]{2}{*}{$p$-value } \\
\hline & ERAS & Standard & & \\
\hline Nombre des patients (\%) & $27(60)$ & $18(40)$ & $45(100)$ & \\
\hline $45(100)$ & & & & 0,4 \\
\hline Masculin & $24(53,3)$ & $14(31,2)$ & $38(84,5)$ & \\
\hline Féminin & $3(6,6)$ & $4(8,9)$ & $7(15,5)$ & \\
\hline $\operatorname{ATCDS}(\%)$ & & & & 0,03 \\
\hline Sans & $21(46,7)$ & $9(20)$ & $30(66,7)$ & \\
\hline HTA & $1(2,2)$ & $2(4,4)$ & $3(6,6)$ & \\
\hline Diabète & $0(0)$ & $4(8,8)$ & $4(8,8)$ & \\
\hline IDM & $0(0)$ & $1(2,2)$ & $1(2,2)$ & \\
\hline Maladie cérébro-vasculaire & $1(2,2)$ & $0(0)$ & $1(2,2)$ & \\
\hline Embolie pulmonaire & $2(4,4)$ & $2(4,4)$ & $4(8,8)$ & \\
\hline $\mathrm{BPCO}$ & $1(2,2)$ & $0(0)$ & $1(2,2)$ & \\
\hline HTA-Diabète & $1(2,2)$ & $0(0)$ & $1(2,2)$ & \\
\hline Score de Charlson (\%) & & & & 0,05 \\
\hline 0 & $20(44,4)$ & $9(20)$ & $29(64,4$ & \\
\hline 1 & $4(8,8)$ & $9(20)$ & $14(28,8$ & \\
\hline 2 & $2(4,4)$ & $0(0)$ & $2(4,4)$ & \\
\hline Type de dérivation (\%) & & & & 0,51 \\
\hline Bricker & $22(48,8)$ & $15(33,4)$ & $37(82,2)$ & \\
\hline Urétérostomie cutanée directe & $1(2,2)$ & $2(4,4)$ & $3(6,6)$ & \\
\hline Entérocystoplastie & $4(8,8)$ & $1(2,2)$ & $5(11,1)$ & \\
\hline Voie d'abord (\%) & & & & 1,00 \\
\hline Ouverte & $23(51,1)$ & $16(35,5)$ & $39(86,6)$ & \\
\hline laparoscopique & $4(8,8)$ & $2(4,4)$ & $6(13,3)$ & \\
\hline Type d'intervention (\%) & & & & 0,8 \\
\hline $\mathrm{CPT}$ & $21(46,7)$ & $16(35,5)$ & $37(82,2)$ & \\
\hline Pelvectomie antérieure & $5(11,1)$ & $2(4,4)$ & $7(15,5)$ & \\
\hline NUT-CPT & $1(2,2)$ & $0(0)$ & $1(2,2)$ & \\
\hline \multicolumn{5}{|c|}{ Classification de Clavien- Dindo (\%) } \\
\hline 1 & $2(4,4)$ & $6(13,3)$ & $8(17,7)$ & \\
\hline 2 & $9(20)$ & $11(24,4)$ & $20(44,4)$ & \\
\hline $3 b$ & $0(0)$ & $1(2,2)$ & $1(2,2)$ & \\
\hline \multicolumn{5}{|l|}{ Type de complications (\%) } \\
\hline Anémie & $9(20)$ & $10(22,2)$ & $19(42,2)$ & \\
\hline TVP & $0(0)$ & $1(2,2)$ & $1(2,2)$ & \\
\hline Pariétales & $1(2,2)$ & $2(4,4)$ & $3(6,6)$ & \\
\hline Gastro-intestinales & $1(2,2)$ & $4(8,8)$ & $5(11,1)$ & \\
\hline Fistule rectale & $0(0)$ & $1(2,2)$ & $1(2,2)$ & \\
\hline
\end{tabular}

ATCDS: antécédents; HTA: hypertension artérielle; IDM: infarctus du myocarde; CPT: cystoprostatectomie totale; NUT: néphrourétérectomie totale; TVP: thrombose veineuse profonde. 
Tableau 2: Les variables qualitatives des patients de deux protocoles de notre série.

\begin{tabular}{lcccc}
\hline & \multicolumn{2}{c}{ Protocole } & Total & $p$-value \\
& ERAS & Standard & & 0.2 \\
\hline Age (ans) & $64 \pm 9$ & $60 \pm 12$ & $62.3 \pm 10.3$ & 0.3 \\
Durée d'intervention (heures) & $275.9 \pm 58.1$ & $257.9 \pm 61.7$ & $268.7 \pm 59.6$ & 0.6 \\
Clairance de créatinine (ml/min/1,73 m2) & $84.5 \pm 27.5$ & $88.4 \pm 24.7$ & $86 \pm 26.2$ & 0.17 \\
Hb préopératoire (g/dl) & $11.5 \pm 1.5$ & $12.2 \pm 1.8$ & $11.8 \pm 1.6$ & 0.24 \\
Pertes sanguines (ml) & $441.1 \pm 176.4$ & $516.1 \pm 225.8$ & $471.1 \pm 198.7$ & $<0.0001$ \\
Délai de reprise gaz (heures) & $36.4 \pm 13.3$ & $69.8 \pm 23$ & $49.8 \pm 24.1$ & $<0.0001$ \\
Délai de première défécation (heures) & $64.6 \pm 18.6$ & $126.6 \pm 40.4$ & $88.5 \pm 41.8$ & 0.003 \\
Echelle visuelle analgésique & & & & 0.007 \\
EVA J0 & $6.4 \pm 0.8$ & $7.4 \pm 1$ & $6.8 \pm 1$ & $<0.0001$ \\
EVA J1 & $5.2 \pm 0.9$ & $6.2 \pm 1.3$ & $5.6 \pm 1.2$ & 0.01 \\
EVA J2 & $4.3 \pm 1.3$ & $5.7 \pm 0.9$ & $4.9 \pm 1.3$ & 0.05 \\
EVA J3 & $3.3 \pm 1.2$ & $4.4 \pm 1.5$ & $3.7 \pm 1.4$ & 0.79 \\
EVA J4 & $2.6 \pm 1.3$ & $3.3 \pm 1.1$ & $2.9 \pm 1.3$ & 0.90 \\
EVA J5 & $1.8 \pm 1.1$ & $1.9 \pm 1$ & $1.9 \pm 1$ & 0.05 \\
EVA J6 & $1.1 \pm 0.7$ & $1 \pm 0.8$ & $1.1 \pm 0.7$ & $10.4 \pm 3.9$ \\
Durée du séjour hospitalier (jours) & $9.4 \pm 3.9$ & $11.7 \pm 3.6$ & & \\
\hline
\end{tabular}

$\mathrm{Hb}$ : hémoglobine

Au total, 45 patients ont été inclus, 18 patients témoin et 27 patients ERAS. La moyenne du séjour hospitalier était de $9.4 \mathrm{j}$ vs $11.7 \mathrm{j} ; p=0.05$ en faveur du groupe ERAS (Figure 1).

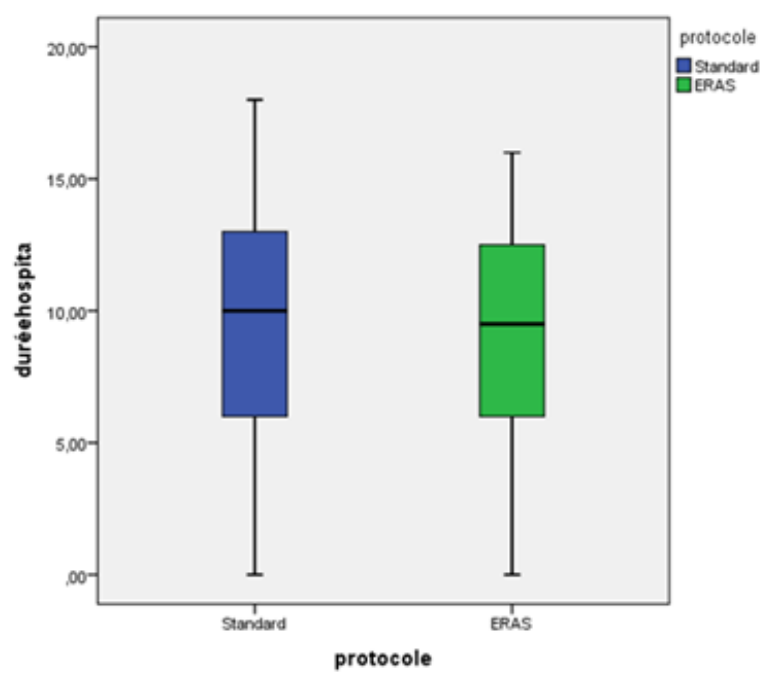

Fig. 1: Comparaison de la durée totale d'hospitalisation entre les deux protocoles ERAS et Standard selon le modèle de Box-plots.
Le délai de reprise de gaz en heures était plus élevé dans le groupe témoin (69.8 vs 36.4; $p=<0.0001$ ) (Figure 2).

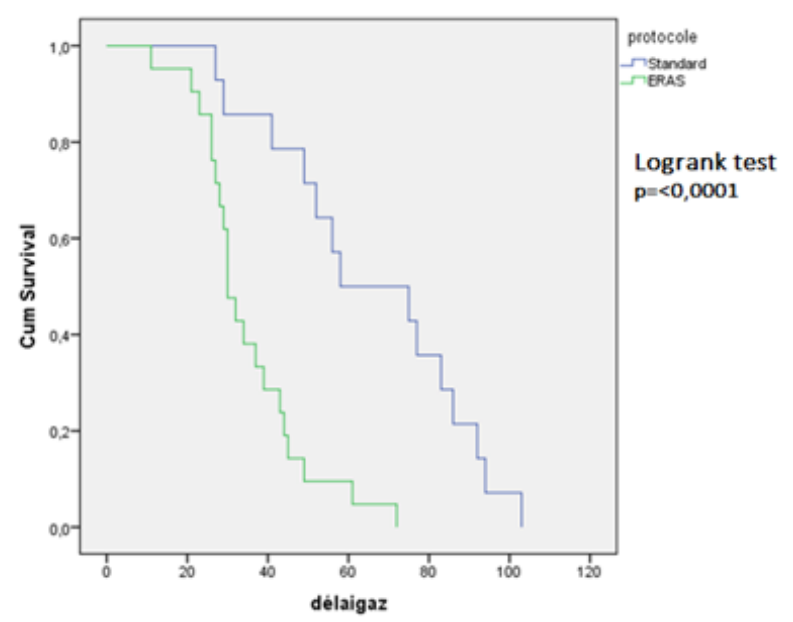

Fig. 2: Comparaison du délai de reprise de gaz entre les deux protocoles ERAS et Standard selon Kaplan-Meier. 
Le délai de la première défécation en heures était moindre dans le groupe ERAS (126.6 vs 64.6; $p=<0.0001$ ) (Figure 3).

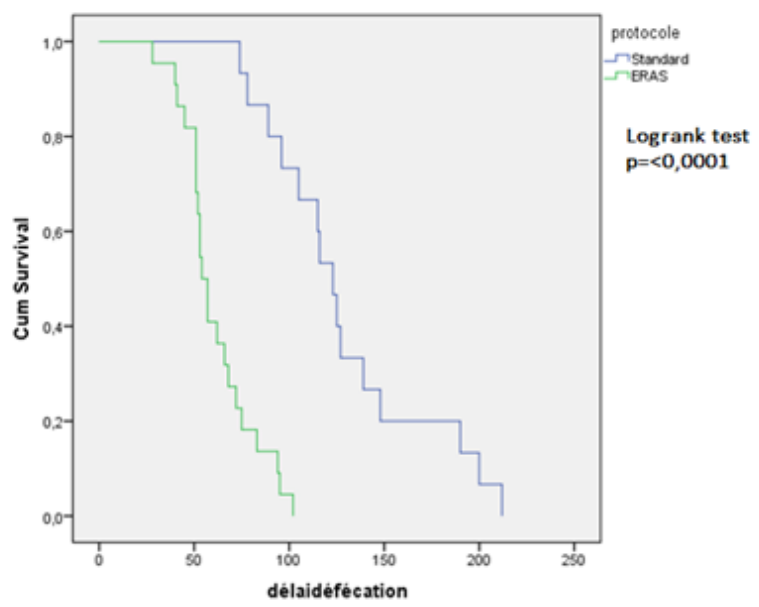

Fig. 3: Comparaison du délai de reprise de défécation entre les deux protocoles ERAS et Standard selon Kaplan-Meier.

Le taux de complications postopératoires générales a été $31 \%$ dans le groupe témoin et de $24.4 \%$ dans le groupe ERAS. Le taux de complications gastro-intestinales était élevé dans le groupe témoin ( $8.8 \%$ vs $2.2 \%)$.

\section{Discussion}

Le concept de réhabilitation précoce ne se limite pas à la période postopératoire mais débute dès la consultation préopératoire avec l'information du patient.

La Récupération améliorée après chirurgie colorectale a fait sa preuve et a montré son efficacité dans la réduction des complications postopératoires ainsi que la durée de l'hospitalisation $^{[6]}$. Malgré les données croissantes en faveur de son efficacité pour des patients subissant une cystectomie radicale, la mise en place des protocoles ERAS dans la pratique d'urologique est toujours limitée ${ }^{[7]}$.

Les résultats périopératoires, tels que le temps opératoire et le taux de transfusion sanguine, sont comparables dans les deux groupes, alors que la dure de séjour médiane est plus courte dans le groupe ERAS.

Les protocoles Enhanced Recovery After Surgery (ERAS) ont démontré un bénéfice en matière de reprise de transit (1 jour), de durée de séjour hospitalier (0.87 jour), et même une diminution des taux de complications (Risk ratio: 0.85 ) selon les résultats de la méta-analyse de 13 de études publiées par Tyson et al. En décembre $2016^{[8]}$.

Aux États-Unis, Daneshmand et al.$^{[5]}$ ont rapporté une série 110 patients en utilisant le protocole ERAS ils ont conclu à une réduction de la durée de séjour médiane à 4 jours. Au Royaume-Uni, Arumainayagam et al. ${ }^{\left[{ }^{[9]}\right.}$ ont trouvé une diminution de la durée d'hospitalisation médiane d'environ 4 jours. La durée de séjour médiane peut refléter aussi bien la conception des soins dans ces pays que la réhabilitation améliorée.

Notre étude, montre que la RAAC réduit la durée moyenne de séjour hospitalier postopératoire, cette réduction a été corroborée par plusieurs autres séries ${ }^{[5}$, ${ }^{10,11]}$. Ainsi qu'une réduction du délai de reprise du transit gazeux ( 1 jours de moins dans le groupe ERAS) et du délai de reprise du transit solide (2.8 jour de moins dans le groupe ERAS).

$\mathrm{Au}$ Royaume-Uni, les patients ne paient pas pour les soins de santé à ce titre, il peut y avoir une réticence à une sortie rapide. Aux États-Unis, les séjours hospitaliers excessivement coûteux incitent les patients à rentrer chez eux ou à s'offrir des soins infirmiers à domicile moins coûteux ( $16 \%$ dans la cohorte Daneshmand et al..$\left.^{[5]}\right)$.

Les principales perspectives des protocoles RAAC, vont dans le sens de proposer des solutions pour lever les obstacles à la mise en place et la généralisation des protocoles ERAS dans les services d'urologie

Concernant les barrières au changement liées aux soignants: Il parait tout à fait justifié d'effectuer des cours/ réunions aux soignants pour présenter les éventuelles recommandations et la littérature sur les différents éléments du RAAC

Concernant les carbohydrates, il faut améliorer la compliance des patients à ce traitement.

Pour cela il faut s'assurer que les patients comprennent son bénéfice.

Concernant les barrières liées aux patients: L'existence d'une personne dédiée au programme RAAC comme coordonnateur des soins pourrait permettre d'éviter les informations contradictoires et permettrait une meilleure éducation thérapeutique. L'objectif final est de permettre aux patients de s'impliquer davantage dans leur prise en charge et leur programme de soins.

Concernant les barrières liées à l'organisation des soins: Le manque de visibilité peut être pallié par une amélioration de l'affichage qui doit être décidé par les soignants euxmêmes, ensemble. Des rappels doivent être mis en place dans le dossier et dans la chambre du patient.

Notre étude n'est pas dépourvue de limites. Premièrement, le design de l'étude empêche une analyse multivariée complète puisque les différents éléments ERAS ont été utilisés ensemble plutôt que dans différentes permutations. 
Deuxièmement, ces données proviennent d'une étude mono centrique avec une équipe unique et donc la présence d'une courbe d'apprentissage doit être prise en considération lors de l'interprétation des résultats. Troisièmement, l'analgésie péridurale qui est recommandée par les sociétés scientifiques et les experts dans les programmes RAAC, ne faisait pas partie de notre protocole et n'était réalisée que chez trois patients.

En dernier, le manque d'aveuglement du personnel médical et infirmier au statut ERAS dans notre étude comparative pourrait avoir introduit un biais d'observateur dans la collecte de données.

\section{Conclusion}

L'optimisation de la prise en charge périopératoire des cystectomies radicales pour cancer de vessie par l'application d'un programme de réhabilitation précoce a permis de réduire de façon significative la durée de séjour et a tendance à améliorer le taux de complications graves ainsi que le taux d'iléus postopératoires.

\section{Approbation éthique et consentement à participer}

Le comité d'éthique de la faculté de médecine de Rabat nous a donné son accord. Le consentement éclairé à participer à l'étude a été fourni par le patient. Le numéro de référence n'est pas applicable.

\section{Consentement à la publication}

Le patient a donné son consentement éclairé et écrit pour la publication de cet ouvrage.

\section{Disponibilité des données et du matériel}

N'est pas applicable.

\section{Intérêts concurrents}

Les auteurs déclarent n'avoir aucun conflit d'intérêts en relation avec cet article.

\section{Financement}

Pas de financement.

\section{Contributions des auteurs}

IZ était l'investigateur de cette étude, AS, IZ et OY ont analysé et interprété les données des patients concernant le sujet. TK, KE, AK et AIAA ont lu et approuvé le manuscrit final.

\section{Références}

[1] Stein JP, Skinner DG. Radical cystectomy for invasive bladder cancer: long-term results of a standard procedure. World J Urol 2006; 24(3):296-304.

[2] Hautmann RE, de Petriconi RC, Volkmer BG. Lessons learned from 1000 neobladders: the 90-day complication rate. J Urol 2010;184(3): 990-4 [quiz 1235].

[3] Shabsigh A, Korets R, Vora KC, et al. Defining early morbidity of radical cystectomy for patients with bladder cancer using a standardized reporting methodology. Eur Urol 2009; 55(1):164-74.

[4] Novotny V, Hakenberg OW, Wiessner D, et al. Perioperative complications of radical cystectomy in a contemporary series. Eur Urol 2007; 51(2):397-402.

[5] Daneshmand S, Ahmadi H, Schuckman AK, et al. Enhanced recovery protocol after radical cystectomy for bladder cancer. J Urol 2014; 192(1):50-5.

[6] Varadhan KK, Neal KR, Dejong $\mathrm{CH}$, et al. The enhanced recovery after surgery (ERAS) pathway for patients undergoing major elective open colorectal surgery: a meta-analysis of randomized controlled trials. Clin Nutr 2010; 29(4):434-440

[7] Cerantola Y, Valerio M, Persson B, et al. Guidelines for perioperative care after radical cystectomy for bladder cancer: enhanced recovery after surgery (ERAS(R)) society recommendations. Clin Nutr 2013; 32(6):879-887

[8] Tyson MD, Chang SS. Enhanced recovery pathways versus standard care after cystectomy: a meta-analysis of the effect on perioperative outcomes. Eur Urol 2016; 70:995-1003.

[9] Arumainayagam N, McGrath J, Jefferson KP, et al. Introduction of an enhanced recovery protocol for radical cystectomy. BJU Int 2008; 101:698-701.

[10] Xu W, Daneshmand S, Bazargani ST, et al. Postoperative pain management after radical cystectomy: comparing traditional versus enhanced recovery protocol pathway. J Urol 2015; 194:1209-1213.

[11] Lee CT, Chang SS, Kamat AM, et al. Alvimopan accelerates gastrointestinal recovery after radical cystectomy: a multicenter randomized placebo-controlled trial. Eur Urol 2014; 66:265-272 


\title{
The benefit of the ERAS protocol in the post-operative of radical cystectomy: Analysis of a Moroccan monocentric study
}

\begin{abstract}
Aim: The aim of this study is to evaluate the impact of the implementation of the ERAS protocol after radical cystectomy for bladder cancer.

Material and Methods: This is a prospective single-center comparative study of a control group with a standard protocol combining cystectomies operated for bladder cancer from March 2017 to April 2018, and an ERAS group. The judgment criteria evaluated were the time to gas recovery, the first defecation, the length of hospital stay.

Results: A total of 45 patients were included, 18 control patients and 27 ERAS patients. The average hospital stay was 9.4 days vs. 11.7 days; $p=0.05$ in favor of the ERAS group. The time to gas recovery in hours was higher in the control group (69.8 vs $36.4 ; p=<0.0001)$. The time to first defecation in hours was less in the ERAS group (126.6 vs $64.6 ; p=<0.0001)$. The rate of general postoperative complications was $31 \%$ in the control group and $24.4 \%$ in the ERAS group.

Conclusion: The application of the ERAS protocol made it possible to reduce the length of hospital stay and tends to reduce the rate of postoperative ileus.
\end{abstract}

Keywords: ERAS; cystectomy; post-operativ. 